\title{
Plasma chromogranin $A$ in patients with sporadic gastro-entero-pancreatic neuroendocrine tumors or multiple endocrine neoplasia type 1
}

\author{
M Peracchi, D Conte, C Gebbia, C Penati, S Pizzinelli, M Arosio ${ }^{1}$, S Corbetta ${ }^{1}$ and A Spada ${ }^{1}$ \\ Department of Medical Sciences and ${ }^{1}$ Institute of Endocrine Sciences, University of Milan, Ospedale Maggiore IRCCS, Padiglione Granelli, \\ Via F Sforza 35, 20122, Milan, Italy \\ (Correspondence should be addressed to M Peracchi; Email: maddalena.peracchi@unimi.it)
}

\begin{abstract}
Objective: As circulating chromogranin A $(\mathrm{CgA})$ has been claimed to be the best general neuroendocrine marker so far available, we evaluated the usefulness of $\mathrm{CgA}$ determination in the clinical assessment of patients with sporadic gastro-entero-pancreatic neuroendocrine tumors (GEP NETs) or multiple endocrine neoplasia type 1 (MEN 1).

Design and methods: Plasma CgA levels were measured using a commercial enzyme-linked immunosorbent assay in 61 patients with sporadic GEP NET and in 25 with MEN 1 including 16 with GEP NET. Controls were 50 healthy volunteers, 46 patients with pituitary adenoma and 35 patients with primary hyperparathyroidism.

Results: The cutoff value for CgA established in our healthy subjects (as mean+2 S.D.) was $20 \mathrm{U} / \mathrm{l}$. CgA levels were above the normal range in 71/77 patients with sporadic or MEN 1-related GEP NETs $(92 \%)$, in four out of nine MEN 1 patients without GEP NETs (44\%), and only in 22/81 control patients with pituitary or parathyroid disease (27\%). Furthermore, CgA levels of over 100 U/l occurred in 36/77 patients with GEP NETs $(47 \%)$ and only in one patient with a non-functioning pituitary adenoma. In the patients with GEP NETs, both tumor burden and secretory activity affected $\mathrm{CgA}$ levels, and successful surgical resection was associated with markedly decreased $\mathrm{CgA}$ values. Conclusions: Plasma CgA was confirmed to be a reliable marker for GEP NETs. Moreover, in MEN 1 patients the finding of very high $\mathrm{CgA}$ levels strongly suggests the presence of a GEP NET, as both primary hyperparathyroidism and pituitary adenomas rarely cause marked $\mathrm{CgA}$ increases.
\end{abstract}

European Journal of Endocrinology 148 39-43

\section{Introduction}

Chromogranin A $(\mathrm{CgA})$ is a $49 \mathrm{kDa}$ monomeric, hydrophilic, acidic glycoprotein that is widely expressed in neuroendocrine cells and constitutes one of the most abundant components of secretory granules (1). It is coreleased with peptidic hormones, biogenic amines and neuropeptides during exocytosis $(2,3)$.

$\mathrm{CgA}$ immunohistochemical detection is a main step in the diagnosis of neuroendocrine tumors (NETs) (3). Also, circulating CgA levels have been claimed to be a useful marker for these tumors, with a high specificity and a sensitivity ranging from 27 to $81 \%$ depending on their origin $(2-8)$. Furthermore, there is evidence that $\mathrm{CgA}$ plasma levels correlate well with tumor burden (4-6).

The highest $\operatorname{CgA}$ values have been found in patients with gastro-entero-pancreatic (GEP) NETs $(4,6)$, and, interestingly, the so-called non-functioning tumors also frequently retain the ability to secrete significant amounts of $\operatorname{CgA}(3,4,9)$. However, most studies have been performed in patients with sporadic GEP NETs, although it is well known that these tumors can occur as a part of multiple endocrine neoplasia syndrome type 1 (MEN 1), which also includes parathyroid and pituitary tumors. Nevertheless, data on the plasma $\operatorname{CgA}$ pattern in patients with this syndrome are scanty $(8,10)$, and the available studies have not evaluated patients with sporadic forms of primary hyperparathyroidism (HPT) or pituitary tumors, although high plasma CgA levels have sometimes been reported in these diseases $(2,11,12)$.

The aim of this study was to evaluate the usefulness of plasma $\mathrm{CgA}$ determination in the clinical assessment of patients with sporadic GEP NET or MEN 1. Results were compared with those of healthy subjects, patients with sporadic primary HPT, and patients with sporadic pituitary tumors. 


\section{Subjects and methods}

Five groups of subjects participated in this study, which was approved by the local ethics committee.

\section{Control}

This group consisted of 50 healthy volunteers, recruited among medical staff and acquaintances of patients, 23 men and 27 women, aged $18-76$ years, mean 45.3 years.

\section{Sporadic primary HPT}

This group consisted of 35 untreated patients, 11 men and 24 women, aged $30-75$ years, mean 62.1 years. Twelve patients had parathyroid hyperplasia at the subsequent surgery and 23 parathyroid adenoma.

\section{Sporadic pituitary tumors}

This group consisted of 46 untreated patients, 18 men and 28 women, aged 32-66 years, mean 54.3 years. Of these, 21 had prolactinoma, 15 acromegaly, five adrenocorticotropin (ACTH)-secreting adenoma, four non-functioning adenoma and one thyrotropin (TSH)-secreting adenoma.

\section{MEN 1}

This group consisted of 25 patients, 11 men and 14 women, aged 17-79 years, mean 46.0 years. All but two patients had HPT. Twelve had pituitary adenoma, and 16 GEP NET (gastrinoma in eight cases, insulinoma in two and non-functioning pancreatic tumor in six, including one with high pancreatic polypeptide and one with high motilin plasma levels). One patient with gastrinoma had gastric carcinoids and four patients, three with gastrinoma and one with insulinoma, had liver metastasis. Nine patients had previously been parathyroidectomized and four had been hypophysectomized. No patient was taking somatostatin analogs at the time of the study.

\section{Sporadic GEP NETs}

This group consisted of 61 untreated patients, 28 men and 33 women, aged $18-81$ years, mean 54.6 years. Of these, 32 had pancreatic tumor (gastrinoma in seven cases, vipoma in four, somatostatinoma in three, calcitoninoma in two, insulinoma in one, ACTH-secreting tumor in one, and non-functioning tumor in 14), 19 had midgut carcinoid and 10 had gastric carcinoid (seven with hypergastrinemia due to chronic atrophic gastritis and three with normogastrinemia). Liver and/or lymph node metastasis was present in 13 patients with pancreatic tumors and in 15 with midgut carcinoids.
Thirteen patients with GEP NETs, four with MEN 1 and nine with sporadic tumor, all without metastasis, were re-evaluated 1 month after tumor resection.

Patients with renal failure or severe hepatic dysfunction were excluded from the study to avoid spuriously elevated CgA levels $(3,8)$.

Venous blood samples were drawn into tubes containing EDTA, between 0800 and $1000 \mathrm{~h}$, after an overnight fast. The samples were centrifuged at $4{ }^{\circ} \mathrm{C}$ and plasma was separated and stored at $-30{ }^{\circ} \mathrm{C}$ until assayed.

$\mathrm{CgA}$ was measured with an enzyme-linked immunosorbent assay purchased from Dako A/S (Glostrup, Denmark). The 95\% confidence limit of detection was $2.1 \mathrm{U} / \mathrm{l}$. The intra- and interassay coefficients of variations were $4.2 \%$ and $6.8 \%$, respectively. As conflicting data have been reported on day-to-day $\mathrm{CgA}$ variation, with values ranging from 0 to $113 \%(10)$, we performed a pilot study measuring CgA levels on two consecutive days in 15 healthy subjects (mean CgA 10.5 U/l) and 20 GEP NET patients (mean CgA 291.7 U/l). Mean day-to-day variation (10) was $10.1 \%$ (range $0.5-31.9 \%$ ) in healthy subjects and $10.4 \%$ (range $1.3-30 \%$ ) in GEP NET patients. Thereafter, only a single sample for $\mathrm{CgA}$ assay was taken in all subjects.

Results are given as medians and interquartile ranges. Statistical analysis was performed using Kruskal-Wallis test followed by Mann-Whitney test with Bonferroni's correction for multiple comparisons. The Wilcoxon test was employed for paired comparisons. Linear regression analysis was performed using log-transformed data. A $P$ value $<0.05$ was considered statistically significant.

\section{Results}

The results are summarized in Table 1 and Fig. 1. The upper reference limit for $\mathrm{CgA}$ defined as 2 S.D. above the mean level of our normal subjects was $20 \mathrm{U} / \mathrm{l}$. Plasma CgA levels were significantly higher than

Table 1 Plasma CgA levels in healthy subjects, patients with primary HPT, pituitary adenomas, MEN 1, sporadic carcinoids and pancreatic NETs. Data are given as medians and interquartile ranges.

\begin{tabular}{lcl}
\hline Diagnosis & No. of cases & Plasma CgA levels (U/I) \\
\hline Healthy subjects & 50 & $12.3(9.3-15.3)$ \\
Primary HPT & 35 & $16.0(11.0-26.9)$ \\
Pituitary adenomas & 46 & $12.7(10.0-19.3)$ \\
MEN 1 & 25 & $35.3(21.0-239.0)^{a, b, c}$ \\
Carcinoids & 29 & $90.0(42.6-551.0)^{\mathrm{a}}$ \\
Pancreatic NETs & 32 & $93.4(25.5-300.0)^{\mathrm{a}}$ \\
\hline
\end{tabular}

${ }^{a} P<0.001$ vs healthy subjects; ${ }^{\text {b}} \mathrm{NS}$ vs carcinoids and pancreatic NETs ${ }^{\mathrm{c}} P<0.01$ vs primary HPT and pituitary adenomas. Statistical analysis was performed using Kruskal-Wallis test followed by Mann-Whitney test with Bonferroni's correction for multiple comparisons. 


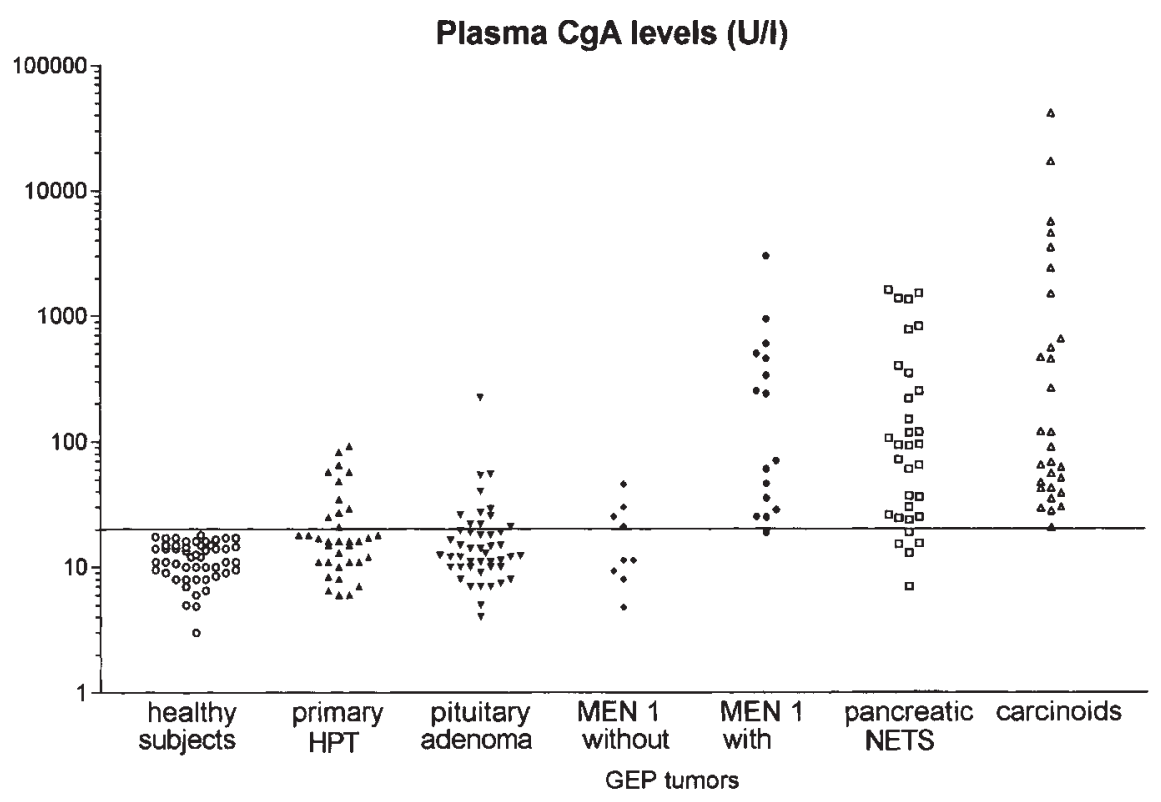

Figure 1 Plasma CgA levels in the various groups of subjects studied. The results are plotted logarithmically to accommodate extreme values. The unbroken line indicates the cutoff $(20 \mathrm{U} / \mathrm{l})$ defined as 2 S.D. above the mean value obtained in our healthy subjects.

those of healthy subjects in patients with MEN 1 syndrome and in patients with sporadic GEP NETs, but not in patients with sporadic primary HPT or pituitary tumors (Table 1). However, in these two latter patient groups we observed $\mathrm{CgA}$ values ranging from 20 to $100 \mathrm{U} / \mathrm{l}$ in 11 and 10 patients, respectively. Also, one patient with non-functioning pituitary tumor had a CgA level of $221 \mathrm{U} / \mathrm{l}$ (Fig. 1). Therefore, arbitrarily, $\mathrm{CgA}$ levels of 20-100 U/l were considered as moderately high and those of over $100 \mathrm{U} / \mathrm{l}$ as very high.

As shown in Fig. 1, CgA levels were elevated in all carcinoid patients. Values were very high in 14 patients (13 with midgut carcinoid and one with gastric carcinoid and hypergastrinemia). CgA levels were normal in five of the 32 patients with sporadic pancreatic tumor (one with somatostatinoma and four with non-functioning tumor), moderately high in 13 (one with insulinoma, two with somatostatinoma, three with vipoma and seven with non-functioning tumor), and very high in 14 (one with ACTH-secreting tumor, one with vipoma, two with calcitoninoma, three with non-functioning tumor and seven with gastrinoma).

In the MEN 1 group, all patients with GEP NET except one with insulinoma had elevated CgA levels. Moderately high values were observed in seven (two with gastrinoma, five with non-functioning tumor) and very high values in eight (one with nonfunctioning tumor, one with insulinoma, six with gastrinoma). Of the nine patients without GEP NET, four (one with HPT, three with HPT and pituitary adenoma) had moderately high CgA values.
In the patients with sporadic pancreatic endocrine tumors or MEN 1 with GEP NETs, plasma CgA levels were significantly higher in the 28 cases of functioning than in the 20 of non-functioning neoplasias (median and interquartile range: $244.5(67.5-690)$ vs 36.5 (24.1-93.9) U/l, $P=0.0015$, Mann-Whitney test). In the 15 gastrinoma patients there was a significant correlation between $\log$-transformed $\mathrm{CgA}$ and gastrin plasma levels $\left(r^{2}=0.515, \quad P=0.0026\right) \quad$ (Fig. 2), whereas a similar correlation was not found in the patients with hypergastrinemia due to chronic atrophic gastritis and gastric carcinoids. Moreover, in patients

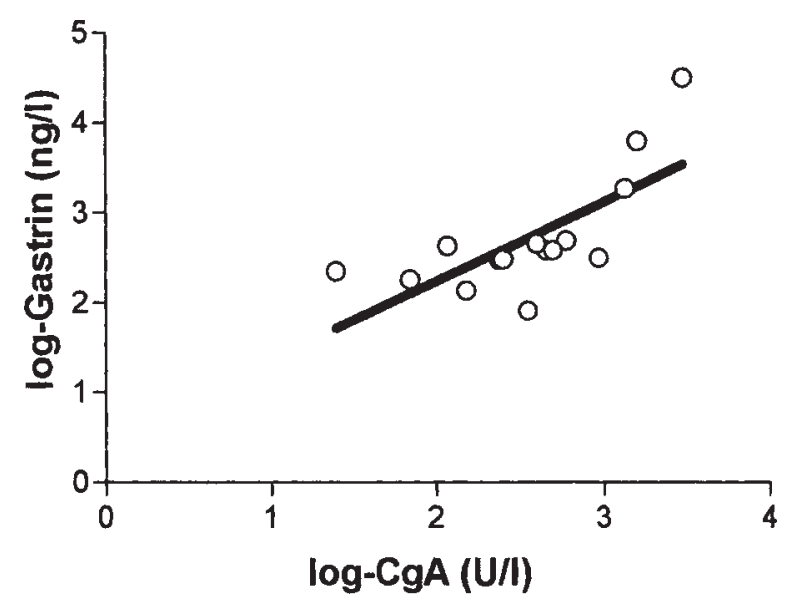

Figure 2 Relation between log-transformed plasma $\mathrm{CgA}$ and gastrin levels in 15 patients with gastrinoma $(y=0.468+0.883 x$; $\left.r^{2}=0.515 ; P=0.0026\right)$. 
with midgut carcinoids there was a significant correlation between log-transformed plasma $\mathrm{CgA}$ and urinary 5-hydroxyindolacetic acid (5-HIAA) levels $\left(r^{2}=0.580, P=0.0002\right)$ (Fig. 3).

In the sporadic or MEN 1-related forms of GEP NET, the 32 patients with metastatic disease had significantly higher $\operatorname{CgA}$ values than the 45 without metastasis, the medians and interquartile ranges being, respectively, $600.5(93.4-1533)$ and 55.6 (29.4-106) U/l, $P<0.0001$ (Mann-Whitney test).

In 13 patients successful surgical treatment of GEP NET was associated with a marked reduction in CgA levels $(120(65-250)$ vs $26(12.8-34.2) \mathrm{U} / \mathrm{l}$, $P=0.0002$, Wilcoxon test).

Finally, the sensitivity and specificity of $\mathrm{CgA}$ assay for GEP NETs were calculated using the healthy subjects and patients with sporadic primary HPT or pituitary tumor as a reference population. Taking $20 \mathrm{U} / \mathrm{l}$ as the cutoff, the sensitivity was $92 \%$ (95\% CI, 84-97\%) and the specificity $83 \%$ (95\% CI, 76-89\%), whereas taking $100 \mathrm{U} / \mathrm{l}$ as the cutoff gave a sensitivity of $47 \%$ $(95 \%$ CI, 35-58) and a specificity of $99 \%$ (95\% CI, 96-100).

\section{Discussion}

In agreement with previous studies $(2,4-8,10)$ our findings clearly demonstrate that abnormally high plasma CgA levels are a characteristic feature of patients with GEP NETs in both sporadic and MEN 1-related forms. The highest $\mathrm{CgA}$ values occurred in patients with metastatic disease, and successful surgical treatment of patients without metastasis tended to normalize the $\operatorname{CgA}$ levels. These observations confirm indirectly that the tumor burden is a major determinant for CgA elevations in GEP NETs (4-6). Indeed, a significant positive correlation between circulating $\mathrm{CgA}$ levels and tumor burden has already

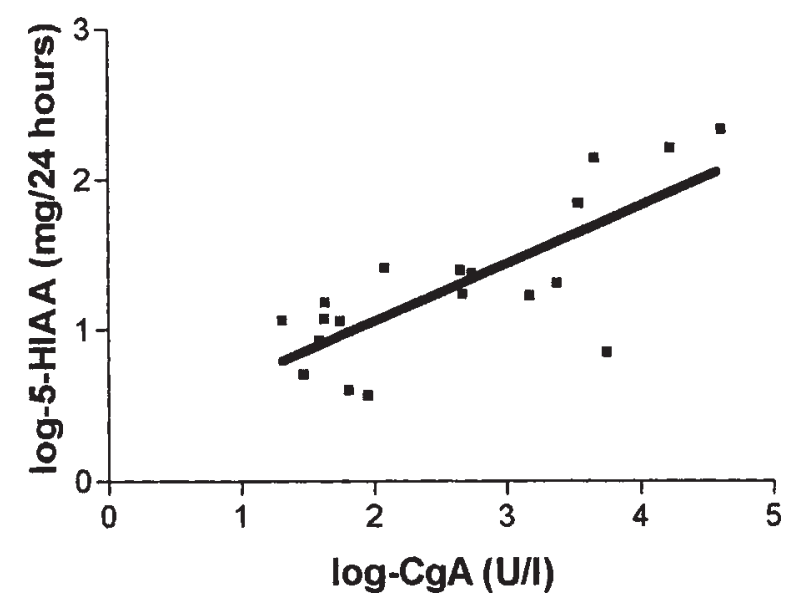

Figure 3 Relation between log-transformed plasma $\mathrm{CgA}$ and urinary 5-HIAA levels in 19 patients with midgut carcinoids $\left(y=0.2866+0.386 x ; r^{2}=0.580 ; P=0.0002\right)$. been demonstrated in patients with carcinoids $(4,5)$ and other NETs (6).

There is also evidence that tumor secretory activity may be another important determinant for $\operatorname{CgA}$ levels, although elevated values have also been found in non-functioning neuroendocrine neoplasias (6). In our study, CgA levels were significantly higher in patients with functioning tumors than in patients with non-functioning tumors. Moreover, plasma $\mathrm{CgA}$ levels correlated significantly with urinary 5-HIAA levels in our patients with midgut carcinoids and with plasma gastrin levels in those with gastrinoma. Similar correlations have already been reported by Nobels et al. (4) and Baudin et al. (6) in carcinoid patients and by Goebel et al. (13) in gastrinomas. Data on gastrinomas warrant consideration. High circulating $\operatorname{CgA}$ levels have frequently been found in gastrinoma patients without any clear correlation with the disease extent $(4,8,13,14)$. It has therefore been suggested that elevations in CgA levels in patients with Zollinger-Ellison syndrome may be due to hyperplasia of the gastric enterochromaffine-like cells caused by chronic hypergastrinemia (14). However, our results do not support this interpretation as we found no correlation between $\mathrm{CgA}$ and gastrin plasma levels in patients with gastric carcinoid and hypergastrinemia due to chronic atrophic gastritis. Moreover, Goebel et al. (13) demonstrated recently that gastrinoma resection significantly reduced CgA levels in patients with Zollinger-Ellison syndrome.

Overall, our data agree with the notion of Nobels et al. (4) that the finding of markedly elevated $\mathrm{CgA}$ levels is highly suggestive of the presence of a NET. However, a major aim of this study was also to assess the diagnostic value of plasma $\mathrm{CgA}$ assay in patients with MEN 1. Therefore, in addition to patients with sporadic and MEN 1-related GEP NETs, we evaluated MEN 1 patients without biochemical and imaging evidence of GEP tumors, and patients with sporadic forms of pituitary adenoma or primary HPT.

There was no significant difference in median plasma CgA levels between healthy subjects and patients with sporadic pituitary tumor or primary HPT, though values above the normal range occurred in 11/46 patients with pituitary adenoma $(24 \%)$ and in $11 / 35$ patients with primary HPT (31\%). Increased circulating $\operatorname{CgA}$ levels have previously been reported in $11-33 \%$ of patients with pituitary adenoma, both secreting and non-functioning $(11,12)$. On the other hand, only two conflicting studies have been published so far on patients with primary HPT. O'Connor and Deftos (2), in their preliminary report, described abnormally high $\mathrm{CgA}$ plasma levels in a series of nine patients with primary HPT, but subsequently Nanes et al. (15) failed to demonstrate a significant increase of $\mathrm{CgA}$ levels in patients with parathyroid adenoma, although elevated values occurred in HPT patients with sporadic or familial parathyroid hyperplasia and in those with 
MEN 1 and Zollinger-Ellison syndrome. In this context it is worthy of note that in our group of patients with sporadic HPT, 12 had parathyroid hyperplasia but only three of them had elevated $\mathrm{CgA}$ levels. Moreover, we also observed moderately high $\mathrm{CgA}$ values in four MEN 1 patients without GEP NET, all with primary HPT and three with pituitary adenoma. Similar results have been reported by Grandberg et al. (10) in their series of MEN 1 patients free from pancreatic involvement. Therefore, the mechanisms governing circulating $\mathrm{CgA}$ levels in patients with primary HPT need further clarification (15).

In all MEN 1 patients with GEP NET except one with insulinoma, we found increased $\mathrm{CgA}$ levels, although very high values (over $100 \mathrm{U} / \mathrm{l}$ ) occurred only in eight patients, most of whom had gastrinoma and/or liver metastasis. These results agree with those of Grandberg et al. (10) and of Tommasetti et al. (8). In the latter series, the percentage of MEN 1 patients with $\mathrm{CgA}$ elevations (53\%) was, however, lower than ours $(94 \%)$, but some of the patients had previously undergone pancreatic surgery (8). Furthermore, neither of these studies evaluated MEN 1 patients with metastatic GEP NET.

The sensitivity and specificity of CgA assay for GEP NETs in both sporadic and MEN 1-related forms clearly depend on the cutoff values chosen and the reference populations $(4,8)$. In establishing cutoff levels, the potential day-to-day variability of plasma CgA (10) should be always taken into consideration, although in our study $\operatorname{CgA}$ values on 2 consecutive days were relatively stable. Moreover, abnormally high $\mathrm{CgA}$ levels occur in patients with renal insufficiency $(3,8)$, and serum creatinine levels should be measured in all subjects with raised $\mathrm{CgA}$ values. Also, slightly elevated $\mathrm{CgA}$ plasma levels may be found in patients with severe liver failure (3), in some patients with inflammatory bowel disease (10), and in some non-endocrine tumors as many of them contain scattered neuroendocrine cells (3, 4). Lastly, somatostatin analog treatment spuriously decreases CgA levels (8).

In conclusion, our study confirms that plasma $\mathrm{CgA}$ is a reliable marker in patients with sporadic and MEN 1related GEP NETs, although it should not be employed in a previously undiagnosed patient as a screening test. However, in MEN 1 patients, the finding of very high $\mathrm{CgA}$ levels is usually indicative of the presence of a GEP NET, whereas moderately high values should prompt other biochemical and imaging studies, and serial $\operatorname{CgA}$ assays are probably helpful in the followup of these patients.

\section{Acknowledgements}

The present study was partially supported by research grants from Ministero dell'Università e della Ricerca
Scientifica (MURST 40\%), from University of Milan (ex MURST 60\%) and from Associazione Amici della Gastroenterologia del Padiglione Granelli, Milan, Italy.

\section{References}

1 Deftos LJ. Chromogranin A: its role in endocrine function and as an endocrine and neuroendocrine tumor marker. Endocrine Reviews $199112181-187$

2 O'Connor DT \& Deftos LJ. Secretion of chromogranin A by peptide producing endocrine neoplasms. New England Journal of Medicine $19863141145-1151$.

3 Nobels FRE, Kwekkeboom DJ, Boullon R \& Lamberts SWJ. Chromogranin A: its clinical value as marker of neuroendocrine tumors. European Journal of Clinical Investigation $1998 \mathbf{2 8}$ 431-440.

4 Nobels FRE, Kwekkeboom DJ, Coopmans W, Schoenmakers CHH, Lindemans J, De Herder WW et al. Chromogranin A as a serum marker for neuroendocrine neoplasia: comparison with neuronspecific enolase and the $\alpha$-subunit of glycoprotein hormones. Journal of Clinical Endocrinology and Metabolism $1997 \mathbf{8 2}$ $2622-2628$.

5 Janson ET, Holmberg L, Stridsberg M, Eriksson B, Theodorsson E, Wilander E et al. Carcinoid tumors. Analysis of prognostic factors and survival in 301 patients from a referral center. Annals of Oncology 19978 685-690.

6 Baudin E, Gigliotti A, Ducreux M, Ropers J, Comoy E, Sabourin JC et al. Neuron-specific enolase and chromogranin A as markers of neuroendocrine tumours. British Journal of Cancer $1998 \mathbf{7 8}$ 1102-1107.

7 Öberg K, Janson ET \& Eriksson B. Tumour markers in neuroendocrine tumours. Italian Journal of Gastroenterology and Hepatology 199931 (Suppl 2) S160-S162.

8 Tommasetti P, Migliori M, Simoni P, Casadei R, De Iasio R, Corinaldesi R et al. Diagnostic value of plasma chromogranin A in neuroendocrine tumors. European Journal of Gastroenterology and Hepatology 200113 55-58.

9 Sobol RE, Memoli V \& Deftos LJ. Hormone-negative chromogranin-A positive endocrine tumors. New England Journal of Medicine $1989320444-447$.

10 Grandberg D, Stridsberg M, Seensalu R, Eriksson B, Lundqvist G, Öberg K et al. Plasma chromogranin A in patients with multiple endocrine neoplasia type 1. Journal of Clinical Endocrinology and Metabolism $1999842712-2717$.

11 Deftos LJ, O'Connor DT, Wilson CB \& Fitzgerald PA. Human pituitary tumors secrete chromogranin-A. Journal of Clinical Endocrinology and Metabolism 198968 869-872.

12 Nobels FR, Kwekkeboom DJ, Coopmans W, Hoekstra R, De Herder WW, Bouillon R et al. A comparison between the diagnostic value of gonadotropins, alpha-subunit, and chromogranin A and their response to thyrotropin-releasing hormone in clinically nonfunctioning, alpha-subunit-secreting and gonadotroph pituitary adenomas. Journal of Clinical Endocrinology and Metabolism 199377 $784-789$.

13 Goebel SU, Serrano J, Yu F, Gibril F, Venzon DJ \& Jensen RT. Prospective study of the value of serum chromogranin A or serum gastrin levels in the assessment of the presence, extent or growth of gastrinomas. Cancer 199985 1470-1483.

14 Stabile BE, Howard TJ, Passarro E \& O'Connor DT. Source of plasma chromogranin A elevation in gastrinoma patients. Archives of Surgery $1990 \mathbf{1 2 5} 451-453$.

15 Nanes MS, O'Connor DT \& Marx SJ. Plasma chromogranin-A in primary hyperparathyroidism. Journal of Clinical Endocrinology and Metabolism $198969950-955$.

Received 18 June 2002

Accepted 9 October 2002 